\title{
Energy-flux vector in anisotropic turbulence: application to rotating turbulence
}

\author{
Naoto Yokoyama ${ }^{1, \dagger} \uparrow$ and Masanori Takaoka ${ }^{2}$ \\ ${ }^{1}$ Department of Mechanical Science and Bioengineering, Osaka University, Toyonaka 560-8531, Japan \\ ${ }^{2}$ Department of Mechanical Engineering, Doshisha University, Kyotanabe 610-0394, Japan
}

(Received 16 March 2020; revised 31 July 2020; accepted 4 October 2020)

Energy flux plays a key role in analyses of energy-cascading turbulence. In isotropic turbulence, the flux is given by a scalar as a function of the magnitude of the wavenumber. On the other hand, the flux in anisotropic turbulence should be a geometric vector that has a direction as well as a magnitude, and depends not only on the magnitude of the wavenumber but also on its direction. The energy-flux vector in anisotropic turbulence cannot be uniquely determined in a way used for the isotropic flux. In this work, introducing two ansatzes, net locality and efficiency of nonlinear energy transfer, we propose a way to determine the energy-flux vector in anisotropic turbulence using the Moore-Penrose inverse. The energy-flux vector in strongly rotating turbulence is demonstrated based on the energy transfer rate obtained by direct numerical simulations. It is found that the direction of the energy-flux vector is consistent with the prediction of the weak turbulence theory in the wavenumber range dominated by inertial waves. However, the energy flux along the critical wavenumbers predicted by the critical balance in the buffer range between the weak turbulence range and the isotropic Kolmogorov turbulence range is not observed in the present simulations. This discrepancy between the critical balance and the present numerical results is discussed and dissipation is found to play an important role in the energy flux in the buffer range.

Key words: rotating turbulence, wave-turbulence interactions, waves in rotating fluids

\section{Introduction}

One of the most important subjects in turbulence research is how energy, which is provided by external force and dissipated by viscosity, is transferred among scales via nonlinear interactions. In Kolmogorov turbulence, the energy cascades from large-scale eddies to small-scale ones via nonlinear interactions. In weak-wave turbulence, the energy is transferred via nonlinear resonant interactions among waves.

The assumption of the weak nonlinearity that the linear time scale is much smaller than the nonlinear time scale is violated at small or large wavenumbers in almost all

$\dagger$ Email address for correspondence: n.yokoyama@mail.dendai.ac.jp

\$ Present address: Department of Mechanical Engineering, Tokyo Denki University, Adachi, Tokyo 120-8551, Japan. 
wave turbulence systems (Biven, Nazarenko \& Newell 2001; Newell, Nazarenko \& Biven 2001; Biven, Connaughton \& Newell 2003). In this case, weak-wave turbulence and strong turbulence coexist (Vinen \& Niemela 2002; Yokoyama \& Takaoka 2014; Meyrand et al. 2018). According to the conjecture of the critical balance (Goldreich \& Sridhar 1995; Nazarenko 2011; Nazarenko \& Schekochihin 2011), the energy is considered to be transferred along the wavenumbers at which the linear wave period is comparable with the eddy turnover time of the isotropic Kolmogorov turbulence in the buffer range between the wavenumber ranges of the weak-wave turbulence and the isotropic Kolmogorov turbulence. The conjecture is being eagerly tested using numerical simulations (TenBarge \& Howes 2012; Ghim et al. 2013; Meyrand, Galtier \& Kiyani 2016; Meyrand et al. 2018).

The scale-by-scale energy cascade is often investigated using energy flux. The constancy of the energy flux in wavenumber space is intensively examined as a corollary of the energy cascade in homogeneous isotropic turbulence (HIT). While the Kolmogorov theory predicts scaling properties, the quantitative feature of the flux for sufficiently large Reynolds numbers has been examined theoretically and numerically.

However, the energy flux in anisotropic turbulence is less elucidated because the analytical expression for the flux is not known, in contrast to HIT. While the flux can be treated as a scalar in HIT, it should be treated as a vector in anisotropic turbulence even if it is homogeneous in real space. In this paper, the definition of the energy-flux vector is proposed, and it is applied to rotating turbulence.

Let us consider a homogeneous anisotropic turbulence system which has one distinguishing direction, say the $z$ direction, and is statistically isotropic in the $x$ and $y$ directions perpendicular to the $z$ direction. It is convenient to investigate the energy transfers in the $k_{\perp}-k_{\|}$plane, where $k_{\perp}=\left|\boldsymbol{k}_{\perp}\right|=\left(k_{x}^{2}+k_{y}^{2}\right)^{1 / 2}$ and $k_{\|}=\left|k_{z}\right|$. In figure 1, the energy flux expected in rotating turbulence is schematically shown. Rotating turbulence is a typical turbulence system where the weak-wave turbulence of inertial waves and the isotropic Kolmogorov turbulence of eddies as well as the two-dimensional columnar vortex coexist (Clark di Leoni et al. 2014; Yokoyama \& Takaoka 2017). In the wavenumber range where the linear period of the inertial wave is shorter than the eddy turnover time, the weak turbulence of the inertial waves is dominant (WT in figure 1). According to the weak turbulence theory, the resonant interactions among the inertial waves transfer energy to waves that have small scales perpendicular to the rotational axis without changing the scales parallel to the rotational axis (Galtier 2003; Bellet et al. 2006; Yarom \& Sharon 2014). On the other hand, the isotropic energy transfer due to the isotropic Kolmogorov turbulence appears in the wavenumber range where the Coriolis period is longer than the eddy turnover time (Mininni, Rosenberg \& Pouquet 2012), i.e. the wavenumber is larger than the Zeman wavenumber $k_{\Omega}$ (KT in figure 1). However, there is no concrete theory that quantitatively gives the energy transfer in the buffer range (green in figure 1). The critical balance predicts the isotropisation due to the redistribution of energy that is transferred anisotropically to the buffer range. If the local energy-flux vectors can be obtained, the arrows of the energy flux in the buffer range are added to figure 1.

The energy flux in HIT is defined as the flux going through a sphere with radius $|\boldsymbol{k}|=k$. To evaluate the energy flux in anisotropic turbulence, the fluxes going through a cylindrical surface with a radius $\left|\boldsymbol{k}_{\perp}\right|=k_{\perp}$ and through planes with $\left|k_{z}\right|=k_{\|}$have been used as a simple tool (Lindborg 2006; Scott \& Arbic 2007; Deusebio et al. 2014). The conical energy flux, which evaluates the flux going through the surface of a cone in wavenumber space, was also proposed by Sharma, Verma \& Chakraborty (2019). The perpendicular energy flux, for example, is obtained by integration over all the parallel wavenumbers, and it corresponds to the flux going through a line parallel to the parallel-wavenumber 


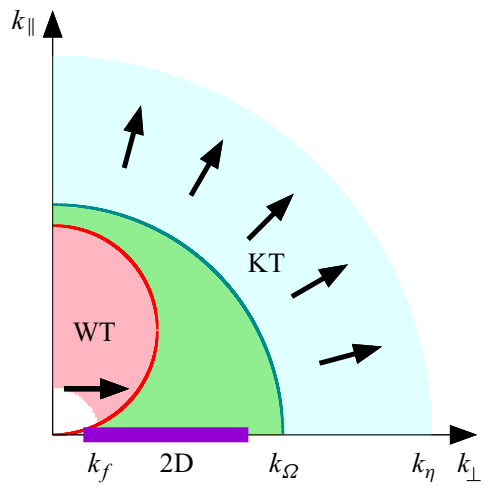

FIGURE 1. Schematic energy flux in rotating turbulence. The wavenumbers of the two-dimensional vortex are indicated by the thick purple line on the $k_{\perp}$ axis. The wavenumber ranges of the weak-wave turbulence, the buffer and the isotropic Kolmogorov turbulence are, respectively, coloured red, green and blue.

axis in figure 1. These integrated energy fluxes inevitably consist of contributions from multiple turbulence ranges: the weak-wave turbulence range, the buffer range and the isotropic Kolmogorov turbulence range. Thus, the local energy flux is expected to be identified to see the paths of energy transfer.

The locality of the net energy transfer in wavenumber space is required for the concept of the flux to be reasonable. The locality is one of the major concepts naturally assumed in the cascading theory, originating from Richardson's poetical note (Richardson \& Lynch 2007) and elaborated by Kolmogorov theory (Kolmogorov 1941). The locality as well as the correction of some aspects of Kolmogorov theory have been widely studied in the literature, starting with the pioneering work of Kraichnan $(1959,1971)$. It should be noted that the non-local energy transfer should be distinguished from the energy transfer in a non-local triad that is a flat triad (Waleffe 1992, 1993). The sweeping effect, in which small-scale eddies are advected by a large-scale eddy, does not make the net non-local energy transfer (Ohkitani \& Kida 1992). In fact, Sharma et al. (2019) reported that the net energy transfer is mainly local in rotating turbulence when the triad interactions are considered as one-to-one interactions. According to the net locality, diffusion models given by partial differential equations in wavenumber space are often employed in weak turbulence (Hasselmann et al. 1985; Dyachenko et al. 1992; Zakharov, L'vov \& Falkovich 1992; Galtier et al. 2019) and in Kolmogorov turbulence (Leith 1967; Connaughton \& Nazarenko 2004). Diffusion models are also applied to anisotropic turbulence systems (Matthaeus, Oughton \& Zhou 2009; Galtier \& Buchlin 2010).

In this paper, the energy-flux vector is obtained using the Moore-Penrose inverse, to identify the direction of the energy flux in anisotropic turbulence. The organisation of this paper is as follows. The procedure for obtaining the energy-flux vector is proposed, and it is verified by examining the energy-flux vector in HIT in $\S 2$. The energy-flux vectors in rotating turbulence are presented in $\S 3$. The validity of the proposed vector in anisotropic turbulence is examined by comparing it with the weak turbulence theory. The energy flux for a more commonly used external forcing is shown in detail. It is consistent with the weak turbulence theory in the wavenumber range where the energy spectrum agrees with the weak turbulence theory, but is observed to be inconsistent with that predicted by the critical balance in the buffer range in this simulation. In $\S 4$, the reason for the 
inconsistency between the fluxes in the present simulation and in the critical balance is clarified. The last section is devoted to a summary.

\section{Formulation of energy-flux vector}

In this section, the energy-flux vector is proposed by introducing two ansatzes. The procedure for numerically obtaining the energy-flux vector is described with a concrete example, which is rotating turbulence. Rotating turbulence is a typical homogeneous anisotropic turbulence system that has been extensively investigated, and can be an appropriate testbed to examine the proposed idea, since it involves different kinds of turbulence (see figure 1). In addition, it is easy to examine the direction of the energy-flux vectors in the range of the inertial-wave turbulence.

\subsection{Integrated energy fluxes}

The governing equations for the velocity $\boldsymbol{u}$ of rotating turbulence in incompressible fluid are the Navier-Stokes equation with the Coriolis term and the divergence-free condition:

$$
\begin{gathered}
\frac{\partial \boldsymbol{u}}{\partial t}+(\boldsymbol{u} \cdot \nabla) \boldsymbol{u}+2 \boldsymbol{\Omega} \times \boldsymbol{u}=-\nabla p+v \nabla^{2} \boldsymbol{u}+\boldsymbol{f}, \\
\boldsymbol{\nabla} \cdot \boldsymbol{u}=0,
\end{gathered}
$$

where the centrifugal force is included in the pressure $p$. The rotation vector $\boldsymbol{\Omega}=\boldsymbol{\Omega} \boldsymbol{e}_{z}$ is assumed to be constant. The governing equations of isotropic turbulence are the same as (2.1) but $\boldsymbol{\Omega}=\mathbf{0}$. The kinematic viscosity is expressed by $v$. The external force $f$ is Gaussian white. Under the periodic boundary condition, the governing equation (2.1) is rewritten in wavenumber space as

$$
\begin{aligned}
& \frac{\partial \boldsymbol{u}_{k}}{\partial t}=-\left(\boldsymbol{I}-\frac{\boldsymbol{k} \otimes \boldsymbol{k}}{k^{2}}\right) \cdot\left(2 \boldsymbol{\Omega} \times \boldsymbol{u}_{k}+\mathrm{i} \sum_{\boldsymbol{k}_{1}+\boldsymbol{k}_{2}=k}\left(\boldsymbol{u}_{\boldsymbol{k}_{1}} \cdot \boldsymbol{k}_{2}\right) \boldsymbol{u}_{k_{2}}\right)-v k^{2} \boldsymbol{u}_{k}+\boldsymbol{f}_{\boldsymbol{k}}, \\
& \boldsymbol{k} \cdot \boldsymbol{u}_{\boldsymbol{k}}=0 \text {. }
\end{aligned}
$$

Energy is transferred among wavenumbers via nonlinear interactions which come from the advection term in (2.2a). The energy transfer rate for a wavenumber $\boldsymbol{k}$ via the nonlinear interactions among three-wavenumber modes is symbolically written as

$$
T_{k}=\left\langle\left.\frac{\partial E_{k}}{\partial t}\right|_{N L}\right\rangle=\sum_{k_{1}, k_{2}} \mathcal{T}\left(\boldsymbol{u}_{k} ; \boldsymbol{u}_{k_{1}}, \boldsymbol{u}_{k_{2}}\right)
$$

where $\boldsymbol{u}_{k}$ and $E_{k}=\left|\boldsymbol{u}_{k}\right|^{2} / 2$ are, respectively, the velocity and the energy of the wavenumber $\boldsymbol{k}$ and $\langle\cdot\rangle$ represents the ensemble average. Note that the energy transfer rate $T_{k}$ is statistically equal to the difference between energy input by the external force and the energy dissipation rate in statistically steady states. The triad interaction function $\mathcal{T}\left(\boldsymbol{u}_{\boldsymbol{k}} ; \boldsymbol{u}_{\boldsymbol{k}_{1}}, \boldsymbol{u}_{k_{2}}\right)$ quantifies the energy transfer from or to the wavenumber $\boldsymbol{k}$ via the triad $\boldsymbol{k}+\boldsymbol{k}_{1}+\boldsymbol{k}_{2}=\mathbf{0}$, and

$$
\mathcal{T}\left(\boldsymbol{u}_{k} ; \boldsymbol{u}_{\boldsymbol{k}_{1}}, \boldsymbol{u}_{\boldsymbol{k}_{2}}\right)=-\frac{\mathrm{i}}{4}\left\langle\left(\boldsymbol{k}_{1} \cdot \boldsymbol{u}_{\boldsymbol{k}_{2}}\right)\left(\boldsymbol{u}_{\boldsymbol{k}} \cdot \boldsymbol{u}_{\boldsymbol{k}_{1}}\right)\right\rangle \delta_{k+k_{1}+k_{2}}+\text { c.c. }+(1 \leftrightarrow 2)
$$

in both isotropic turbulence and rotating turbulence studied in this paper. Here, $(1 \leftrightarrow$ 2 ) represents the terms with the subscripts 1 and 2 interchanged in the preceding ones. 
The triad interaction function $\mathcal{T}\left(\boldsymbol{u}_{\boldsymbol{k}} ; \boldsymbol{u}_{\boldsymbol{k}_{1}}, \boldsymbol{u}_{\boldsymbol{k}_{2}}\right)$ is symmetric under the interchange of $\boldsymbol{k}_{1}$ and $\boldsymbol{k}_{2}$, and the energy detailed balance $\mathcal{T}\left(\boldsymbol{u}_{\boldsymbol{k}} ; \boldsymbol{u}_{\boldsymbol{k}_{1}}, \boldsymbol{u}_{\boldsymbol{k}_{2}}\right)+\mathcal{T}\left(\boldsymbol{u}_{\boldsymbol{k}_{1}} ; \boldsymbol{u}_{\boldsymbol{k}_{2}}, \boldsymbol{u}_{\boldsymbol{k}}\right)+\mathcal{T}\left(\boldsymbol{u}_{k_{2}} ; \boldsymbol{u}_{\boldsymbol{k}}\right.$, $\left.\boldsymbol{u}_{\boldsymbol{k}_{1}}\right)=0$ holds. The triad interaction function $\mathcal{T}\left(\boldsymbol{u}_{\boldsymbol{k}} ; \boldsymbol{u}_{\boldsymbol{k}_{1}}, \boldsymbol{u}_{\boldsymbol{k}_{2}}\right)$ is the sum of the energy transfer between $\boldsymbol{k}$ and $\boldsymbol{k}_{1}$ and that between $\boldsymbol{k}$ and $\boldsymbol{k}_{2}$.

To confirm the cascade theory in HIT, the energy flux

$$
P(k)=-\int_{0}^{k} \mathrm{~d} k^{\prime} T\left(k^{\prime}\right)
$$

is usually examined. The isotropic energy transfer rate $T(k)$ is obtained from $T_{k}$ by integration over the solid angle of $\boldsymbol{k}$, and is assumed to be a continuous function of $k=|\boldsymbol{k}|$.

Anisotropic turbulence generally has a distinguishing direction, which is, for example, the direction of the rotational axis in rotating turbulence. The $z$ direction is set to be such a distinguishing direction in this paper. The $x$ and $y$ directions are perpendicular to the distinguishing direction. Suppose that the system statistically has azimuthal symmetry with respect to the $z$ direction, i.e. azimuthal isotropy in the $x-y$ plane. Then, the statistical quantities can be described in the $k_{\perp}-k_{\|}$plane, where $k_{\perp}=\left(k_{x}^{2}+k_{y}^{2}\right)^{1 / 2}$ and $k_{\|}=\left|k_{z}\right|$.

As a natural extension of the isotropic energy flux (2.5) to azimuthally symmetric turbulence, the energy fluxes perpendicular and parallel to the system's distinguishing direction,

$$
\begin{aligned}
& P_{\perp}\left(k_{\perp}\right)=-\int_{0}^{k_{\perp}} \mathrm{d} k_{\perp}^{\prime} \int_{0}^{\infty} \mathrm{d} k_{\|}^{\prime} T\left(k_{\perp}^{\prime}, k_{\|}^{\prime}\right), \\
& P_{\|}\left(k_{\|}\right)=-\int_{0}^{k_{\|}} \mathrm{d} k_{\|}^{\prime} \int_{0}^{\infty} \mathrm{d} k_{\perp}^{\prime} T\left(k_{\perp}^{\prime}, k_{\|}^{\prime}\right),
\end{aligned}
$$

have been used (e.g. Alexakis et al. 2007). The anisotropic energy transfer rate $T\left(k_{\perp}, k_{\|}\right)$is obtained from $T_{k}$ by integration over the azimuthal angle of $\boldsymbol{k}_{\perp}$ and the sign of $k_{z}$. Because $P_{\perp}\left(k_{\perp}\right)$ and $P_{\|}\left(k_{\|}\right)$are, respectively, obtained by integration over $k_{\perp}$ and $k_{\|}$, detailed local structures such as critical balance in wavenumber space cannot be captured directly by these integrated energy fluxes. These energy fluxes, (2.5) and (2.6), are referred to as integrated fluxes in this paper.

\subsection{Minimal-norm energy-flux vector}

To quantitatively investigate the energy-transfer mechanism in anisotropic turbulence, the detailed structure local in wavenumber space of the energy flux needs to be investigated. In this paper, the scalar-valued energy flux defined in HIT is extended to a vector-valued energy flux in anisotropic turbulence. Because of the energy cascade, the definition of the energy flux in HIT (2.5) implicitly assumes the net locality of nonlinear interactions and the local energy conservation in wavenumber space:

$$
T(k)+\frac{\mathrm{d} P(k)}{\mathrm{d} k}=0 .
$$

As an extension to the energy flux in anisotropic turbulence, we assume local energy conservation:

$$
T_{k}+\operatorname{div}_{k} P_{k}=0
$$

where $\operatorname{div}_{k}$ is the divergence operator in wavenumber space and $\boldsymbol{P}_{\boldsymbol{k}}=\left(P_{x \boldsymbol{k}}, P_{y \boldsymbol{k}}, P_{z k}\right)$ is the energy-flux vector. 
In general, the nonlinear interactions due to the advection term contain both local and non-local interactions in wavenumber space. However, when we try to draw the flux as a vector field, we must implicitly consider the flux to represent the local interactions. Therefore, the ansatz of the local energy conservation is a natural consequence from the present purpose of finding the energy-flux vector. The local energy conservation (2.8) can be interpreted as an alternative expression of the net local interactions to the diffusion models where the energy transfer in wavenumber space is approximated by partial differential equations (Matthaeus et al. 2009; Galtier \& Buchlin 2010).

The origin of the anisotropy of the energy flux should be described here based on (2.8). The anisotropy of the energy-flux vector comes from that of the energy transfer rate. In the statistically steady state, the anisotropy of the energy transfer rate can be explained in terms of the energy input due to the external force and the dissipation rate due to the viscous term. The velocity and hence the energy can be statistically anisotropic owing to the system's anisotropy. In rotating turbulence, the anisotropy of the energy is notable at small wavenumbers. Thus, the energy input given by the inner product of the velocity and the external force is anisotropic owing to the anisotropy of the velocity even if the external force is isotropic. The dissipation rate at small wavenumbers is also anisotropic owing to the anisotropy of the energy even if the viscous term and the dissipation rate at large wavenumbers outside of the isotropic Kolmogorov turbulence range are isotropic. The energy-flux vector is determined by the energy transfer rate over the whole of the wavenumber domain. It is similar to the pressure obtained by solving the Poisson equation in real space. Therefore, the energy-flux vector is anisotropic in anisotropic turbulence even if the external force and the viscous term are isotropic. The local energy conservation (2.8) is extended to a discrete formulation that is convenient in the numerical analysis. Let us consider the energy balance in a coarse-grained cell that has side lengths $\Delta k_{x}, \Delta k_{y}$ and $\Delta k_{z}$ in wavenumber space as shown in figure 2 . The energy balance in the $(i, j, k)$ cell is obtained from the local energy conservation (2.8) as

$$
\mathrm{P}_{x}^{i+1 / 2, j, k}-\mathrm{P}_{x}^{i-1 / 2, j, k}+\mathrm{P}_{y}^{i, j+1 / 2, k}-\mathrm{P}_{y}^{i, j-1 / 2, k}+\mathrm{P}_{z}^{i, j, k+1 / 2}-\mathrm{P}_{z}^{i, j, k-1 / 2}=-\mathrm{T}^{i, j, k} .
$$

The energy fluxes incoming to and outgoing from the cell, $\mathbf{P}_{x}^{i \pm 1 / 2, j, k}, \mathrm{P}_{y}^{i, j \pm 1 / 2, k}$ and $\mathbf{P}_{z}^{i, j, k \pm 1 / 2}$, are defined on the cell faces, and the energy transfer rate of the cell $\mathrm{T}^{i, j, k}$ is given as the sum of the energy transfer rates of the wavenumbers in the cell. It is represented in a matrix-vector form as

$$
D \mathrm{P}=-\mathrm{T},
$$

where $\boldsymbol{D} \in \mathbb{R}^{N_{x} N_{y} N_{z} \times\left(3 N_{x} N_{y} N_{z}-N_{x} N_{y}-N_{y} N_{z}-N_{z} N_{x}\right)}$ corresponds to the difference in (2.9) derived from the divergence operator in (2.8), $\mathrm{P} \in \mathbb{R}^{\left(3 N_{x} N_{y} N_{z}-N_{x} N_{y}-N_{y} N_{z}-N_{z} N_{x}\right) \times 1}$ is a solution column vector of the flux and $T \in \mathbb{R}^{N_{x} N_{y} N_{z} \times 1}$ is the column vector of the transfer rates. The numbers of the coarse-grained cells in the $x, y$ and $z$ directions are, respectively, $N_{x}, N_{y}$ and $N_{z}$. The number of components of $\mathrm{P}$ is approximately three times larger than that of T, since $\boldsymbol{P}_{\boldsymbol{k}}$ consists of three components in three-dimensional turbulence while $T_{k}$ is a scalar. Note that the number of components of $P$ is smaller than three times that of $T$, because the energy flux to or from the outer range of the computational domain does not exist.

Obviously, the divergence matrix $\boldsymbol{D}$ has linearly independent rows. Thus, the solution of (2.10) is not unique. Nature often adopts the most efficient way under constraints. For example, the minimal energy state where the Euclidean norm of the velocity vector is minimal is realised under the condition that the vorticity invariants are conserved in Euler flows (Vallis, Carnevale \& Young 1989). The minimal-norm flux proposed here has the minimal Euclidean norm under the condition that the energy transfer rate is provided 


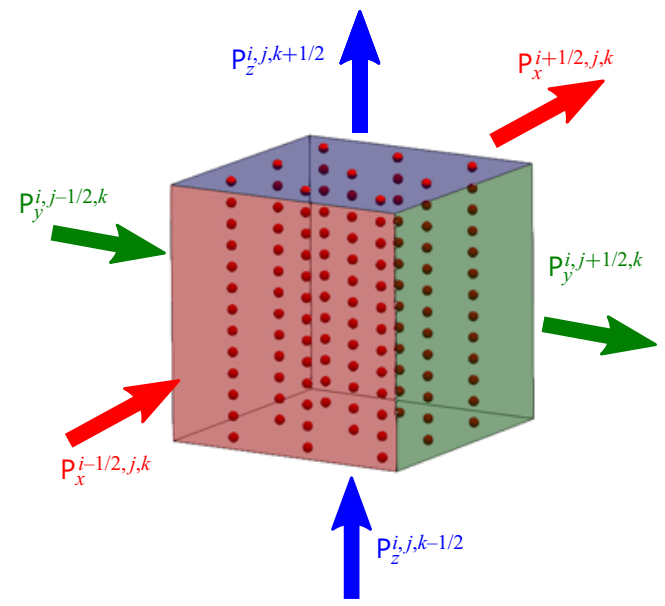

FIGURE 2. Coarse-graining in wavenumber space and local energy balance. The red points represent the wavenumbers at which the energy transfer rates are evaluated.

by nonlinear interactions. The selection of the minimal-norm vector is the least-action principle in this system where the Euclidean norm of the energy-flux vector is considered as an action. We here introduce this principle to uniquely determine energy-flux vectors, the solution of (2.10).

It is the Moore-Penrose inverse, which is a generalised inverse, that can find an appropriate flux vector among the infinite number of solutions. For $\boldsymbol{D}$ having linearly independent rows, the Moore-Penrose inverse is defined as $\boldsymbol{D}^{+}=\boldsymbol{D}^{\mathrm{T}}\left(\boldsymbol{D} \boldsymbol{D}^{\mathrm{T}}\right)^{-1}$. The Moore-Penrose inverse selects the solution as $\mathrm{P}_{*}=-\boldsymbol{D}^{+} \mathrm{T}=\operatorname{argmin}\|\mathrm{P}\|_{2}$ such that (2.10) holds. Namely, $\mathrm{P}_{*}$ selected by the Moore-Penrose inverse has the minimal Euclidean norm among the infinite number of solutions of (2.10).

In addition, the minimal-norm solution $\mathrm{P}_{*}$ is irrotational. Because the divergence of the rotation is 0 and because every null-space component of the matrix having linearly independent rows is orthogonal to any of the row-space components, $\mathrm{P}_{*}$ does not have the null-space solenoidal component. Thus, the use of the Moore-Penrose inverse to (2.10) is equivalent to the assumption that the energy-flux vector is irrotational. The selection of the minimal-norm flux can be interpreted as that the energy transfer is 'efficient' in the sense that the minimal-norm flux excludes local circulations of the energy transfer.

The minimal-norm solution $\mathrm{P}_{*}$ is obtained by numerically solving (2.10) as follows. The energy transfer rate for each wavenumber mode $T_{k}$ in the statistically steady state is obtained in direct numerical simulation (DNS). The column vector of the transfer rates $T$ is composed by coarse-graining of $T_{k}$. Using the generalised minimal residual (GMRES) method, $\left(\boldsymbol{D} \boldsymbol{D}^{\mathrm{T}}\right)^{-1} \mathrm{~T}$ is obtained. For the convergence criterion, the relative error is below $10^{-10}$. The minimal-norm vector $P_{*}$ is obtained by applying $-\boldsymbol{D}^{\mathrm{T}}$ to the vector obtained in the previous step.Once $\mathrm{P}_{*}$ is obtained, the energy-flux vector of the wavenumber $\boldsymbol{k}$ located at the centre of the $(i, j, k)$ cell is given as

$$
\boldsymbol{P}_{\boldsymbol{k}}=\left(\frac{\mathrm{P}_{*}^{i+1 / 2, j, k}+\mathrm{P}_{*}^{i-1 / 2, j, k}}{2 \Delta k_{y} \Delta k_{z}}, \frac{\mathrm{P}_{*}^{i, j+1 / 2, k}+\mathrm{P}_{*}^{i, j-1 / 2, k}}{2 \Delta k_{z} \Delta k_{x}}, \frac{\mathrm{P}_{*}^{i, j, k+1 / 2}+\mathrm{P}_{*}^{i, j, k-1 / 2}}{2 \Delta k_{x} \Delta k_{y}}\right) \text {. }
$$

The energy-flux vector in the system with one distinguishing direction and statistical isotropy in the directions perpendicular to the distinguished direction is reduced to the 


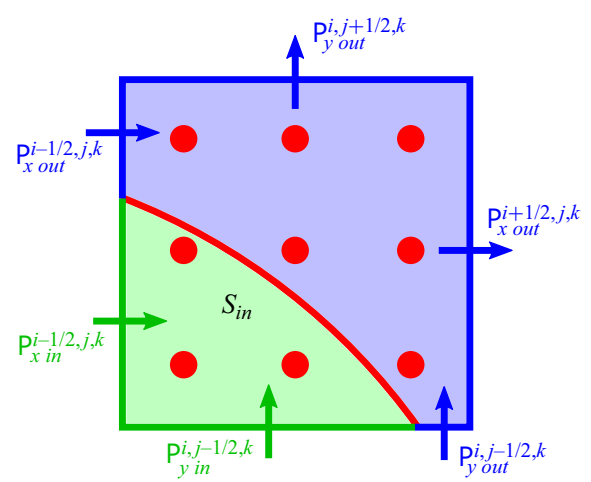

FIGURE 3. Perpendicular energy flux through an arc.

two-dimensional energy-flux vector in the $k_{\perp}-k_{\|}$plane. The perpendicular and parallel components of the energy-flux vector are obtained by averaging over the azimuthal angles and the signs of $k_{z}$. Let us evaluate the perpendicular component of the energy-flux vector going through an arc in a coarse-grained cell represented by the red curve in figure 3 . In this example, the incoming flux $\mathrm{P}_{\perp \text { in }}$ is the sum of the energy fluxes through the sides inside the arc, $\mathrm{P}_{x i n}^{i-1 / 2, j, k}$ and $\mathrm{P}_{y \text { in }}^{i, j-1 / 2, k}$. Here, the flux on the cut-cell edge $\mathrm{P}_{x}^{i-1 / 2, j, k}$, for example, is divided into $\mathrm{P}_{x \text { in }}^{i-1 / 2, j, k}$ and $\mathrm{P}_{x \text { out }}^{i-1 / 2, j, k}$ according to the divided lengths. The outgoing flux $\mathrm{P}_{\perp \text { out }}$ is similarly obtained. The energy flux through the arc is obtained by a weighted average of the incoming and outgoing fluxes as

$$
\mathrm{P}_{\perp \text { arc }}=\frac{\mathrm{P}_{\perp \text { in }} S_{\text {out }}+\mathrm{P}_{\perp \text { out }} S_{\text {in }}}{S_{\text {in }}+S_{\text {out }}}
$$

where $S_{\text {in }}$ and $S_{\text {out }}$, respectively, denote the areas inside and outside the arc and $S_{\text {in }}+S_{\text {out }}=$ $\Delta k_{x} \Delta k_{y}$. The perpendicular component of the energy-flux vector is obtained by averaging over these arcs as well as the sign of $k_{z}$. The parallel component of the energy-flux vector is obtained from the $z$ component of the energy flux by averaging over the azimuthal angles and the signs of $k_{z}$.

\subsection{Energy-flux vector in HIT}

To confirm the consistency of the minimal-norm energy-flux vector with the integrated energy fluxes (2.5) and (2.6), the minimal-norm energy-flux vector is obtained from DNS of the well-known HIT. The DNS is performed with $512^{3}$ grid points in a cubic box whose volume is $(2 \pi)^{3}$. The pseudo-spectral method with aliasing removal by the phase shift is employed to evaluate the nonlinear term, and hence the maximal wavenumber is approximately $512 \sqrt{2} / 3 \approx 240$. The Runge-Kutta-Gill method is used for the time integration. The external force generated by white noise is added in wavenumber space to the wavenumber mode in $k_{f}-1 / 2 \leq|k|<k_{f}+1 / 2$, where the forced wavenumber $k_{f}$ is set to 4 in this simulation.

In the statistically steady state, the nonlinear energy transfer rate for each wavenumber mode $T_{k}$ is obtained. The column vector of the energy transfer rates in the coarse-grained cell with side $\Delta k_{x}=\Delta k_{y}=\Delta k_{z}=\Delta k=3$ is composed, and the solution vector of the energy flux is obtained as the minimal-norm vector. The energy-flux vector is converted to the two-dimensional vector in the $k_{\perp}-k_{\|}$plane using the azimuthal average. The vector 

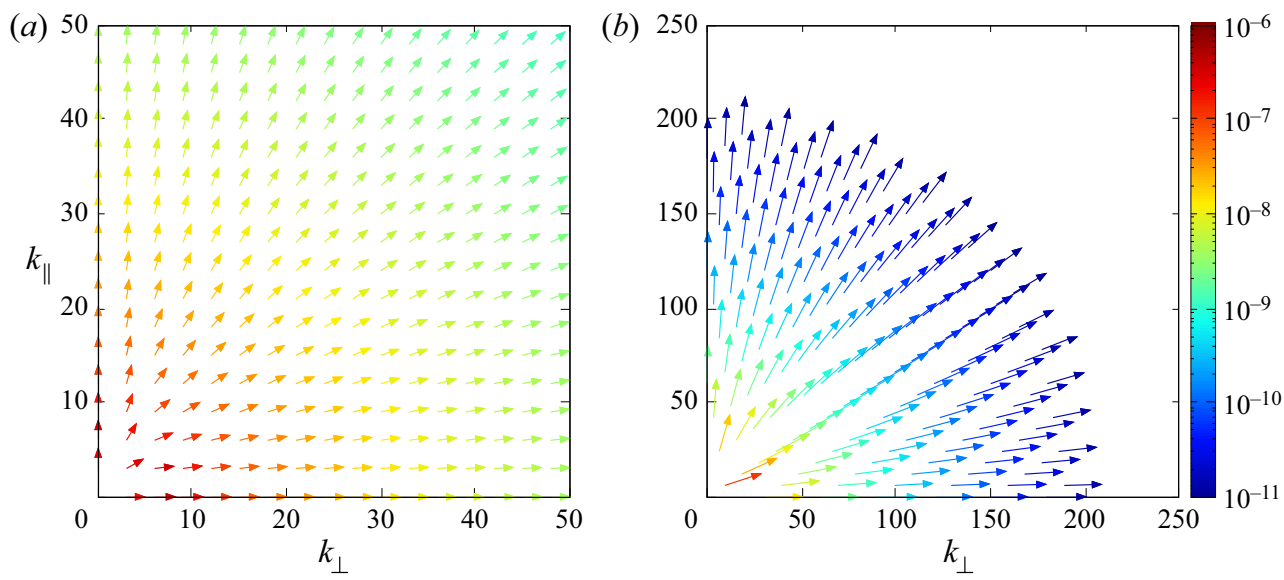

FIGURE 4. Energy-flux vector in HIT $(a)$ in the small-wavenumber range and $(b)$ in the whole computational domain.

in HIT is examined to have only a radial component in the $k_{\perp}-k_{\|}$plane. Once the isotropy of the minimal-norm energy-flux vector in HIT is verified, the direction of the energy flux in anisotropic turbulence can be examined by the minimal-norm vector below.

The two-dimensional energy-flux vectors in HIT are shown in figure 4. The two-dimensional energy-flux vectors are obtained from the three-dimensional energy-flux vectors by averaging over the azimuthal angles and the signs of $k_{z}$ as described in $\$ 2.2$. Figure $4(a)$ is an enlarged view in the small-wavenumber range, while the vectors in the whole computational domain are shown by omitting some vectors for clarity in figure $4(b)$. The energy-flux vectors radiate outward at all wavenumbers as expected by the forward cascade of energy and its isotropy. The magnitudes of the energy-flux vectors are large near the origin, and become small as the magnitudes of the wavenumbers $k=\left(k_{\perp}^{2}+k_{\|}^{2}\right)^{1 / 2}$ become large, because the areas of the spheres on which the flux is evaluated are proportional to $k^{2}$ and energy conservation holds under (2.8).

The direction of the energy flux is quantitatively evaluated to justify the validity of the minimal-norm energy-flux vector. The angle between $\boldsymbol{P}_{\boldsymbol{k}}$ and $\boldsymbol{k}$ is defined as

$$
\theta_{k}=\cos ^{-1} \frac{\boldsymbol{k} \cdot \boldsymbol{P}_{\boldsymbol{k}}}{|\boldsymbol{k}|\left|\boldsymbol{P}_{\boldsymbol{k}}\right|},
$$

and the angle is expected to be 0 in HIT. The mean as well as the standard deviation of the angle in the spherical shell $k-\Delta k / 2 \leq|\boldsymbol{k}|<k+\Delta k / 2$ are shown in figure $5(a)$. The angles are small, that is, the energy-flux vectors are radial at all wavenumbers. In particular, the energy-flux vectors in the inertial subrange are almost completely radial. The small but relatively large angles at small wavenumbers are mainly due to the fluctuation of the random external force. The angles increase near the largest wavenumber owing to the boundary condition of the energy-flux vectors, but the magnitudes of the energy-flux vectors are negligibly small. The isotropy of the energy flux in HIT is successfully validated.

To confirm the consistency of the minimal-norm energy-flux vector with the integrated energy fluxes, integrated energy fluxes are constituted from the energy-flux vectors. The integrated perpendicular flux $P_{\perp}^{v}\left(k_{\perp}\right)$ is obtained by the summation of $P_{x}$ and $P_{y}$ through the arc $(2.12)$ as well as the summation over $k_{z}$. The integrated parallel flux $P_{\|}^{v}\left(k_{\|}\right)$is 

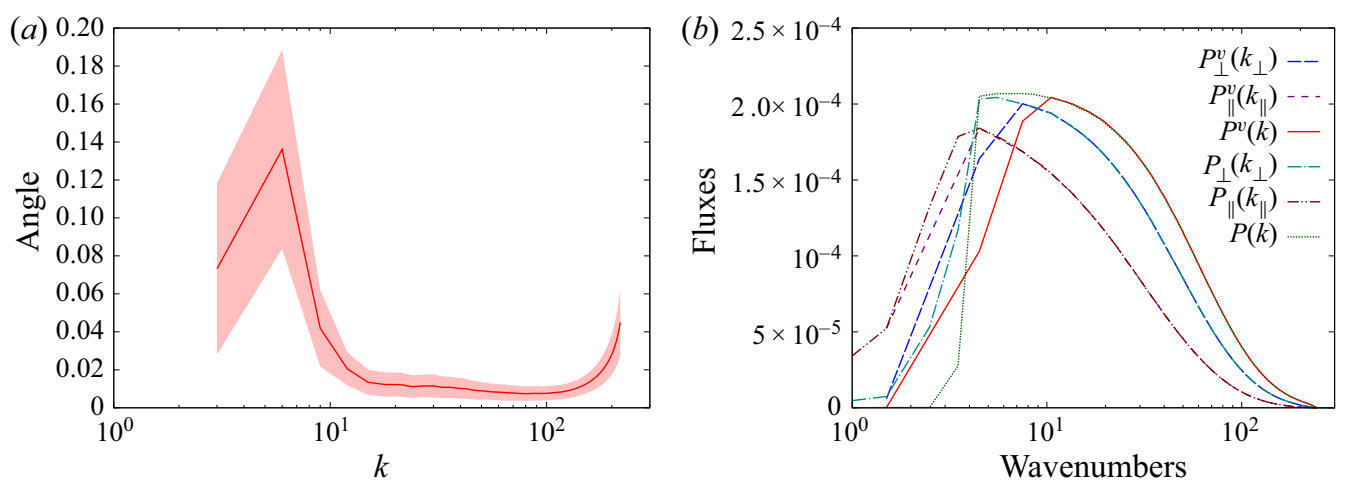

Figure 5. (a) Angle between $\boldsymbol{P}_{\boldsymbol{k}}$ and $\boldsymbol{k}$ measured in radians. The mean and the standard deviation are represented by the solid curve and the shaded region, respectively. (b) Integrated energy fluxes in HIT. The integrated fluxes with the superscript $v, P_{\perp}^{v}, P_{\|}^{v}$ and $P^{v}$, are obtained from the three-dimensional energy-flux vectors, while those without the superscript, $P_{\perp}, P_{\|}$and $P$, are obtained according to (2.5) and (2.6).

obtained by the summation of $P_{z}$ over $k_{x}$ and $k_{y}$ and the signs of $k_{z}$. The isotropic energy flux $P^{v}(k)$ is obtained by averaging the energy fluxes through the inner and outer surfaces of the cells which cover the sphere with radius $k$. These integrated energy fluxes constituted from the energy-flux vectors are compared with the integrated energy fluxes (2.5) and (2.6) in figure 5(b).

The perpendicular energy fluxes, $P_{\perp}^{v}$ and $P_{\perp}$, are almost equal to each other. The difference at small perpendicular wavenumbers $k_{\perp}<6$ comes from the discreteness of the coarse-grained cell during the conversion from $P_{x}$ and $P_{y}$ to $P_{\perp}^{v}$. The conversion does not make a difference at large wavenumbers because the difference between the incoming and outgoing fluxes due to discreteness of the cells is not so large there. Moreover, if the outgoing flux of the cell instead of the weighted average (2.12) is used as the flux to evaluate $P_{\perp}^{v}$, then $P_{\perp}^{v}$ and $P_{\perp}$ are almost equal to each other at small wavenumbers, though $P^{v}$ is then shifted slightly to smaller wavenumber at large wavenumbers. Similarly, the radial energy fluxes, $P^{v}$ and $P$, are almost equal to each other, though a difference at small wavenumbers also emerges owing to the discreteness of the cells. Because $P_{\|}^{v}$ is evaluated exactly on the cell faces, and is not affected by the discreteness of the cells, $P_{\|}^{v}$ and $P_{\|}$ are equal to each other over all wavenumbers within the convergence criterion during the calculation by the GMRES method. Note that $P_{\|}^{v}$ has its value at $k_{\| i}=3(i-1 / 2)$ owing to the coarse-graining with $\Delta k=3$ as described above, while $P_{\|}$has its at $k_{\| j}=j-1 / 2$, where $i$ and $j$ here denote positive integers. Therefore, these energy fluxes obtained from the energy-flux vectors are equal to the integrated energy fluxes except for the difference due to the discreteness of the cells. In this way, the magnitudes of the energy-flux vectors obtained by the Moore-Penrose inverse are quantitatively consistent with the integrated energy fluxes in HIT.

\section{Application to strongly rotating turbulence}

In this section, the energy flux as the minimal-norm solution of the continuity equation of energy is examined in rotating turbulence by comparing the vector with theoretical predictions. Direct numerical simulations of rotating turbulence are performed 
according to (2.2) using the pseudo-spectral method. In the following simulations of rotating turbulence, the periodic box has dimensions of $2 \pi \times 2 \pi \times 8 \pi$ so that $k_{x}, k_{y} \in \mathbb{Z}$ and $k_{z} \in \mathbb{Z} / 4$. Here, a periodic box long in the $z$ direction is used because of the anisotropy at large scales. The non-dimensional numbers that characterise rotating turbulence are the turbulent Reynolds number $R e_{t}=\bar{\varepsilon}^{1 / 3} /\left(\nu k_{f}^{4 / 3}\right)=\left(k_{\eta} / k_{f}\right)^{4 / 3}$ and the rotational Reynolds number $\operatorname{Re}_{\Omega}=\bar{\varepsilon} /\left(\nu \Omega^{2}\right)=\left(k_{\eta} / k_{\Omega}\right)^{4 / 3}$, where $\bar{\varepsilon}$ is the energy dissipation rate and $k_{\eta}$ is the Kolmogorov wavenumber.

\subsection{Theoretical prediction of energy flux in rotating turbulence}

When the nonlinear term, the viscosity and the external force in the governing equation (2.2) are neglected, the linear inviscid equation can be written as

$$
\partial a_{k}^{s_{k}} / \partial t=-\mathrm{i} s_{k} \sigma_{k} a_{k}^{s_{k}}
$$

where the complex amplitude $a_{k}^{s_{k}}$ is defined as $a_{\boldsymbol{k}}^{s_{k}}=\boldsymbol{u}_{\boldsymbol{k}} \cdot \boldsymbol{h}_{\boldsymbol{k}}^{-s_{k}}$ according to the helical-mode decomposition (Waleffe 1993; Smith \& Waleffe 1999; Galtier 2003; Alexakis 2017) and $s_{k}= \pm 1$ denotes the sign of the helicity of the inertial wave. The basis is expressed as $\boldsymbol{h}_{\boldsymbol{k}}^{s_{k}}=\left(\boldsymbol{e}_{1}+\mathrm{i} s_{\boldsymbol{k}} \boldsymbol{e}_{2}\right) / \sqrt{2}$, where $\left(\boldsymbol{e}_{1}, \boldsymbol{e}_{2}\right)=\left(\boldsymbol{e}_{z} \times \boldsymbol{k} /\left|\boldsymbol{e}_{z} \times \boldsymbol{k}\right|, \boldsymbol{k} \times\left(\boldsymbol{e}_{z} \times\right.\right.$ $\left.\boldsymbol{k}) /\left|\boldsymbol{k} \times\left(\boldsymbol{e}_{z} \times \boldsymbol{k}\right)\right|\right)$ for $k_{\perp} \neq 0$, and $\left(\boldsymbol{e}_{1}, \boldsymbol{e}_{2}\right)=\left(\boldsymbol{e}_{x}, \boldsymbol{e}_{y}\right)$ for $k_{\perp}=0$. The linear dispersion relation is given by $\sigma_{k}=2 \Omega k_{z} / k$. The linear inviscid equation (3.1) has the wave solutions $\boldsymbol{u}(\boldsymbol{x}) \propto \boldsymbol{h}_{k}^{s_{k}} \exp \left(\mathrm{i}\left(\boldsymbol{k} \cdot \boldsymbol{x}-s_{\boldsymbol{k}} \sigma_{\boldsymbol{k}} t\right)\right)+$ c.c. called inertial waves.

In the wave-dominant range, the period of the inertial wave is considered to be much shorter than the eddy turnover time, that is, $1 / \sigma_{k} \ll\left(k^{2} \bar{\varepsilon}\right)^{-1 / 3}$ (Clark di Leoni et al. 2014). On the premise of the local nonlinear interaction, Galtier (2003) applied the weak turbulence theory to inertial waves in strongly rotating turbulence, and he found that only a small energy transfer along $\boldsymbol{\Omega}$ is allowed by the resonance condition:

$$
\boldsymbol{k}=\boldsymbol{k}_{1}+\boldsymbol{k}_{2}, \quad s_{k} \sigma_{k}=s_{k_{1}} \sigma_{k_{1}}+s_{k_{2}} \sigma_{k_{2}}
$$

He also discussed the non-locality of the nonlinear interactions, which generates the anisotropy. Waleffe (1993) applied his idea of the instability assumption on the nonlinear energy transfers to predict the anisotropic energy transfer among the wavenumber modes and obtained similar results. The energy flux parallel to the perpendicular wavenumber axis is theoretically expected in the wave-dominant range.

At wavenumbers larger than the Zeman wavenumber, where the Coriolis period is larger than the eddy turnover time, the rotation is negligible at such large wavenumbers. Thus, isotropic Kolmogorov turbulence appears at the larger wavenumbers, and the energy flux is isotropic like that in HIT shown in figure 4.

The energy flux in the buffer range between the weak turbulence range and the isotropic Kolmogorov turbulence range is expected to connect the energy flux parallel to the perpendicular wavenumber axis in inertial-wave turbulence and the isotropic energy flux in isotropic Kolmogorov turbulence. Nazarenko \& Schekochihin (2011) predicted that the energy is transferred along the wavenumbers at which the period of the inertial wave is comparable with the eddy turnover time, after the energy is carried to such wavenumbers by the resonant interactions among inertial waves. That is, the critical balance predicts that the energy is transferred to large $k_{\|}$in the buffer range in figure 1. 

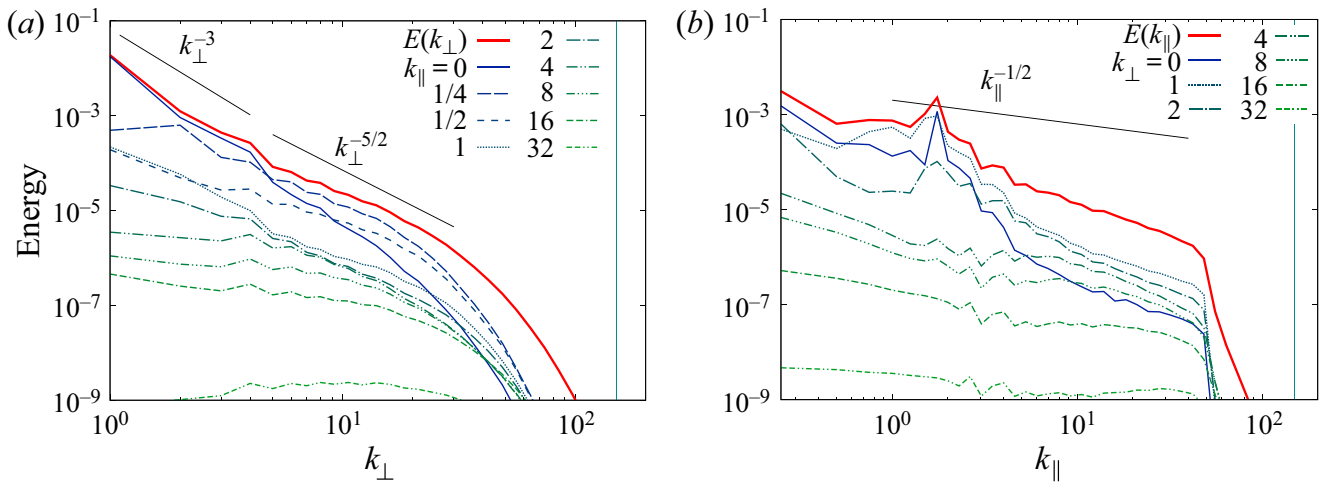

FIGURE 6. Kinetic energy spectra when an external force is applied to small perpendicular wavenumbers $(a)$ for each $k_{\|}$as a function of $k_{\perp}$ and $(b)$ for each $k_{\perp}$ as a function of $k_{\|}$.

\subsection{Minimal-norm energy-flux vector in rotating turbulence}

\subsubsection{Comparison with the weak turbulence theory}

In order to validate the minimal-norm energy-flux vector in anisotropic turbulence, it is compared with the energy flux in the weak turbulence theory. A DNS of rotating turbulence where the external force is applied to the wavenumber modes $k_{\perp} \approx 0$ is performed to compare the energy-flux vectors directly with the prediction of the weak turbulence theory of inertial waves. Here, a random external force is applied to the small perpendicular wavenumber modes $k_{x}, k_{y}=0, \pm 1$ and $\left|k_{z}\right| \leq 50$ in a DNS with $256 \times$ $256 \times 1024$ grid points. In this simulation, the rotational Reynolds number is evaluated as $R e_{\Omega} \approx 0.4$. Although the turbulent Reynolds number is not well defined in this simulation because the forced wavenumbers are widely distributed, the turbulent Reynolds number is considered to be small to investigate the energy flux in weak turbulence. Time averaging to obtain the energy transfer rate $T_{k}$ required in the local energy conservation (2.8) as well as the energy spectra is performed in the statistically steady state.

In the weak turbulence theory, the energy spectrum of the inertial waves is predicted as $E\left(k_{\perp}, k_{\|}\right) \propto k_{\perp}^{-5 / 2} k_{\|}^{-1 / 2}$ (Galtier 2003). To observe the anisotropic spectra, the kinetic energy spectra for each $k_{\|}$as a function of $k_{\perp}$ and for each $k_{\perp}$ as a function of $k_{\|}$are shown in figure 6. The energy spectrum for each $k_{\|}$as a function of $k_{\perp}$, for example, is defined as

$$
E_{k_{\|}}\left(k_{\perp}\right)=\frac{1}{\Delta k_{\perp}} \sum_{k_{\perp}^{\prime}}^{\prime} \frac{1}{\Delta k_{\|}} \sum_{k_{z}^{\prime}}^{\prime} \frac{1}{2}\left\langle\left|\boldsymbol{u}_{k_{\perp}^{\prime}, k_{z}^{\prime}}\right|^{2}\right\rangle,
$$

where the summations $\sum_{k_{\perp}^{\prime}}$ and $\sum_{k_{\|}^{\prime}}^{\prime}$ are, respectively, taken over $k_{\perp}-\Delta k_{\perp} / 2 \leq\left|\boldsymbol{k}_{\perp}^{\prime}\right|<$ $k_{\perp}+\Delta k_{\perp} / 2$ and $k_{\|}-\Delta k_{\|} / 2 \leq\left|k_{z}^{\prime}\right|<k_{\|}+\Delta k_{\|} / 2$, and $\Delta k_{\perp}$ and $\Delta k_{\|}$are the bin widths to obtain the spectrum. The corresponding integrated spectra as a function of $k_{\perp}$

$$
E\left(k_{\perp}\right)=\frac{1}{\Delta k_{\perp}} \sum_{k_{\perp}^{\prime}}^{\prime} \sum_{k_{z}^{\prime}} \frac{1}{2}\left\langle\left|\boldsymbol{u}_{k_{\perp}^{\prime}, k_{z}^{\prime}}\right|^{2}\right\rangle=\int \mathrm{d} k_{\|} E_{k_{\|}}\left(k_{\perp}\right)
$$

are also shown in figure 6.

The perpendicular-wavenumber spectra of the energy are close to $k_{\perp}^{-5 / 2}$ in the wavenumber range $4 \lesssim k_{\perp} \lesssim 30$ and $10 \lesssim k_{\|} \lesssim 30$. Similarly, the parallel-wavenumber 

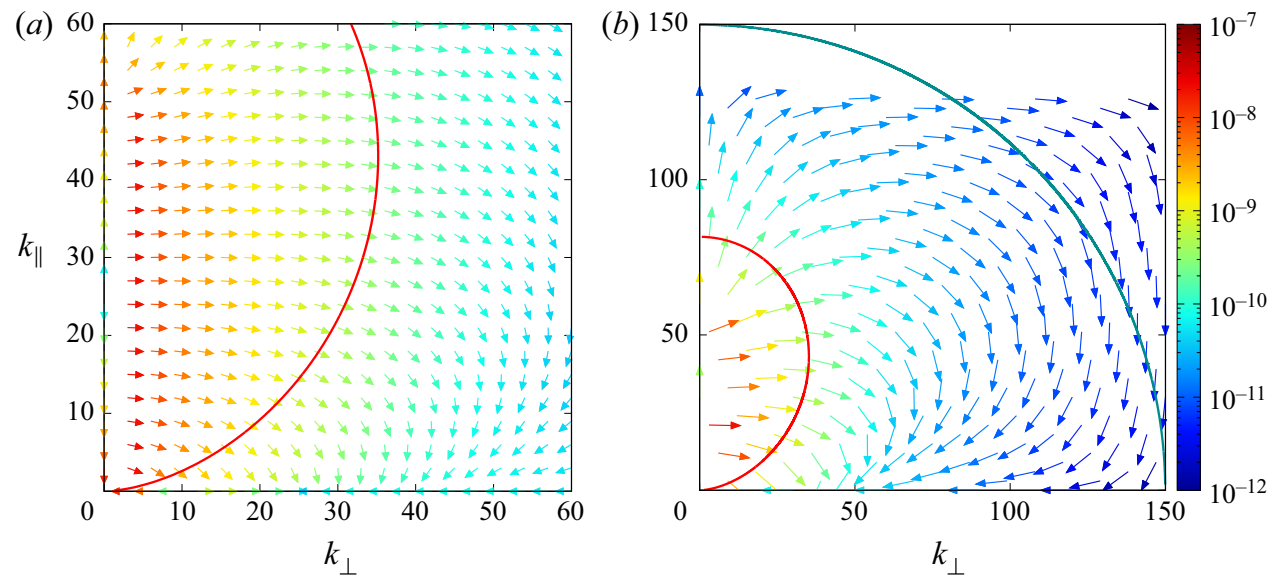

FIGURE 7. Energy-flux vectors when an external force is applied to small perpendicular wavenumbers $(a)$ in the small-wavenumber range and $(b)$ in the whole computational domain. The critical wavenumber and the Zeman wavenumber are, respectively, shown as red and cyan curves.

spectra of the energy are close to $k_{\|}^{-1 / 2}$ in the same wavenumber range. Moreover, the parallel-wavenumber spectra have abrupt drops at $k_{\|} \approx 50$, and the energy injected by the external force is rarely transferred to the large parallel wavenumbers $k_{\|}>50$. This is consistent with the prediction of the weak turbulence theory of inertial waves, in which the resonant interactions transfer the energy only to wavenumbers having the same $k_{\|}$. These spectra demonstrate that the inertial-wave turbulence appears in the wavenumber range $4 \lesssim k_{\perp} \lesssim 30$ and $5 \lesssim k_{\|} \lesssim 30$. The energy spectra show that this DNS is appropriate for comparing the energy-flux vector with the prediction of the weak turbulence theory.

The energy-flux vector in rotating turbulence, the spectra of which are shown in figure 6, is obtained by the procedure described in $\$ 2.2$. The perpendicular and parallel components of the energy-flux vectors are, respectively, obtained from the $x$ and $y$ components and the $z$ components by averaging over the azimuthal angles and the signs of $k_{z}$. The energy-flux vectors in the $k_{\perp}-k_{\|}$plane are shown in figure 7.

Weak-wave turbulence is expected to occur at wavenumbers where the linear wave period $\tau_{w}=\left(2 \Omega k_{\|} / k\right)^{-1}$ is much shorter than the eddy turnover time $\tau_{e}=\left(k^{2} \bar{\varepsilon}\right)^{-1 / 3}$. The critical wavenumber is evaluated to appear at $\tau_{w}=\tau_{e} / 3$ in magnetohydrodynamic turbulence (Meyrand et al. 2016) and stratified turbulence (Yokoyama \& Takaoka 2019). On the other hand, the Coriolis force affects little and isotropic Kolmogorov turbulence appears at the wavenumber range where the Coriolis period $\tau_{\Omega}=(2 \Omega)^{-1}$ is larger than the eddy turnover time $\tau_{e}$. The separation wavenumber of isotropic Kolmogorov turbulence is known as the Zeman wavenumber $k_{\Omega}$. A buffer range should exist between the critical wavenumber and the Zeman wavenumber. The curves representing the critical wavenumber (red) and the Zeman wavenumber (cyan) are shown in figure 7.

The energy-flux vectors at the wavenumber modes $k_{\|} \leq 50$ in the weak turbulence range are almost completely parallel to the $k_{\perp}$ axis, and the energy provided by the external force rarely goes to the modes $k_{\|}>50$. Thus, the wavenumber modes at $k_{\|}>50$ in the weak turbulence range have little energy. The minimal-norm vector can well reproduce 


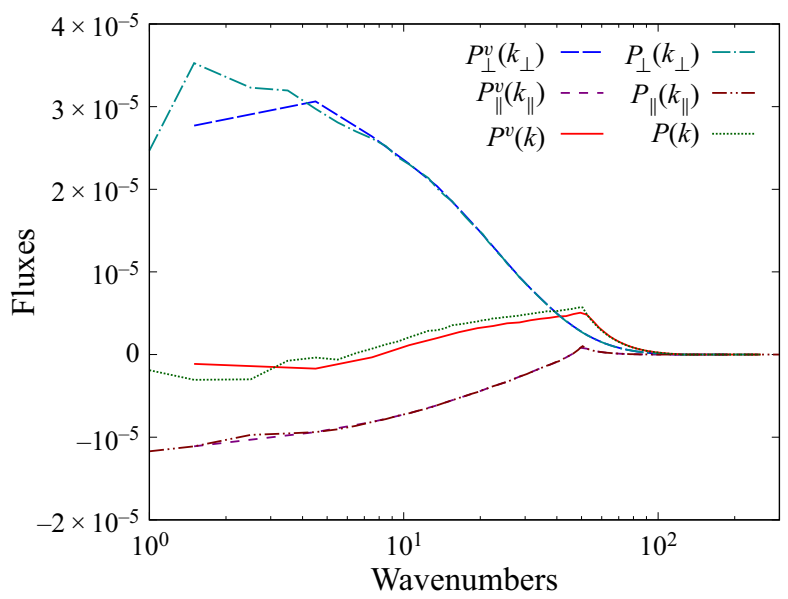

FIGURE 8. Perpendicular, parallel and isotropic integrated fluxes when an external force is applied to small perpendicular wavenumbers. The superscript $v$ denotes the integrated fluxes obtained from the three-dimensional energy-flux vectors. See also caption to figure 5.

the anisotropic energy flux in accordance with the resonant interactions among the inertial waves locally in wavenumber space.

The energy flux in the range where $\tau_{w}<\tau_{e} / 3$ and weak-wave turbulence is expected to exist does not necessarily demonstrate a perpendicular flux. This results from the fact that the wavenumber modes at $k_{\|}>50$ have little energy and the modes in the range are subordinate to the modes at $k_{\|}<50$ having much larger energy.

The energy-flux vectors turn in direction near the wavenumber modes having $\tau_{w}=\tau_{e} / 3$. In this case, the energy flux along the critical wavenumbers that gives the isotropisation of energy in the buffer range is not observed. In fact, the energy transferred via the resonant interactions moves on to the two-dimensional modes $k_{\|}=0$.

The integrated energy fluxes obtained from the three-dimensional energy-flux vectors in the simulation where the external force is applied to the small perpendicular wavenumbers are almost equal to the corresponding integrated energy fluxes as shown in figure 8 . The difference between $P_{\perp}^{v}$ and $P_{\perp}$ at small perpendicular wavenumbers $k_{\perp}<6$ comes from the discreteness of the coarse-grained cell during the conversion from $P_{x}$ and $P_{y}$ to $P_{\perp}^{v}$ as seen in isotropic turbulence (figure 5). The difference between $P^{v}$ and $P$ at $k<50$ where the external force has a direct effect comes from the discreteness of the coarse-grained cell during averaging the energy fluxes over the azimuthal angles or through the inner and outer surfaces of the cells. The negative parallel flux, $P_{\|}<0$, appearing at $k_{\|}<50$ where the external force affects shows that most of the energy provided by the external force goes to small parallel wavenumbers, and is dissipated there.

\subsubsection{Energy flux in DNS with isotropic forcing}

Another DNS where more commonly used external force is employed is performed with $512 \times 512 \times 2048$ grid points. One may think that the resolution is not so high as that in recent high-resolution simulations. However, such high-resolution simulation is not easily performed because a long-time integration is required to obtain the weak inertial-wave turbulence where the energy is transferred by the resonant interactions. 


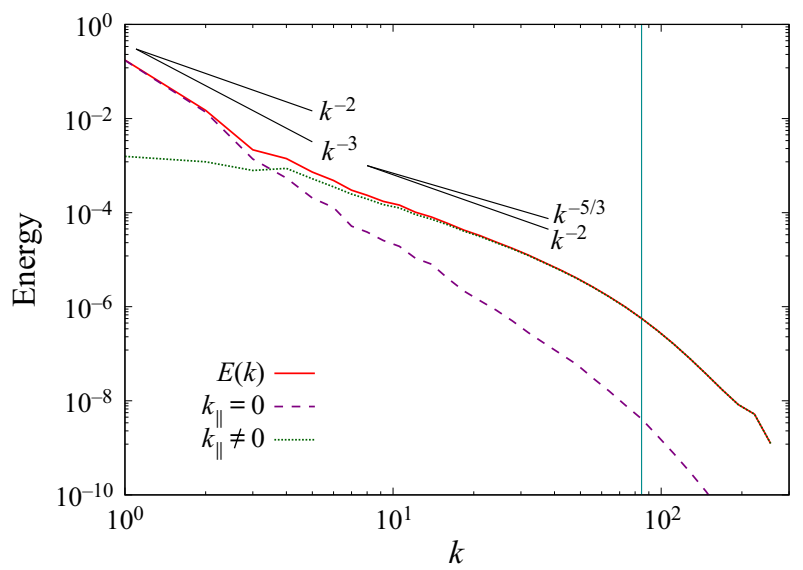

FIGURE 9. Energy spectrum, that given only by two-dimensional modes $\left(k_{\|}=0\right)$ and that given by three-dimensional modes $\left(k_{\|} \neq 0\right)$. The vertical line indicates the Zeman wavenumber.

Three-dimensional three-component random force is added isotropically to the small wavenumbers in $k_{f}-1 / 2 \leq|\boldsymbol{k}|<k_{f}+1 / 2$, where the forced wavenumber $k_{f}$ is set to 4. In this DNS, the turbulent Reynolds number and the rotational Reynolds number are, respectively, evaluated as $R e_{t} \approx 1.6 \times 10^{2}$ and $R e_{\Omega} \approx 2.8$.

The energy spectrum as a function of the norms of wavenumbers obtained in the DNS is shown in figure 9. The energy spectrum at wavenumbers smaller than those of the external force $k_{f} \approx 4$ is as steep as $k^{-3}$. The two-dimensional flows, which are uniform in the direction parallel to the rotational axis, i.e. $k_{\|}=0$, account for a large fraction of the energy at small wavenumbers, though the number of two-dimensional modes is much smaller than that of the three-dimensional modes which have $k_{\|} \neq 0$. In fact, a large-scale columnar vortex is formed. Note that a statistically steady state can be achieved without small-wavenumber drag (Chan, Mitra \& Brandenburg 2012). At wavenumbers larger than $k_{f}$, the energy spectrum is dominated by three-dimensional flows, and is less steep than at small wavenumbers. In this energy spectrum, the isotropic Kolmogorov turbulence cannot be clearly observed, because the Zeman wavenumber is close to the Kolmogorov wavenumber as well as the cutoff wavenumber.

The energy spectra are shown in figure 10. The spectra are close to the prediction of the weak turbulence theory, $E\left(k_{\perp}, k_{\|}\right) \propto k_{\perp}^{-5 / 2} k_{\|}^{-1 / 2}$, in the wavenumber range $4 \lesssim$ $k_{\perp} \lesssim 30$ and $1 / 4 \lesssim k_{\|} \lesssim 4$. The abrupt drops at $k_{\|} \approx 4=k_{f}$ in the parallel-wavenumber spectra indicate that little energy is transferred to the large parallel wavenumbers $k_{\|}>k_{f}$ consistent with the weak turbulence theory. These spectra demonstrate that inertial-wave turbulence appears in the wavenumber range $4 \lesssim k_{\perp} \lesssim 30$ and $1 / 4 \lesssim k_{\|} \lesssim 4$.

The energy-flux vectors in the $k_{\perp}-k_{\|}$plane in isotropically forced rotating turbulence are shown in figure 11 . The energy-flux vectors roughly in the range $k_{\perp} \lesssim 30$ and $k_{\|} \lesssim 4$ are almost parallel to the $k_{\perp}$ axis. The perpendicular flux is consistent with the resonant interactions of inertial waves in the weak turbulence theory. In fact, the range where the perpendicular flux is observed corresponds to the range where the weak inertial-wave spectra are observed in figure 10.

On the other hand, the vector field of the energy flux has a sink at $\left(k_{\perp}, k_{\|}\right) \approx(100,0)$. In this simulation, the energy flux along the critical wavenumbers predicted by the critical balance is not observed. This discrepancy is discussed in the next section. 

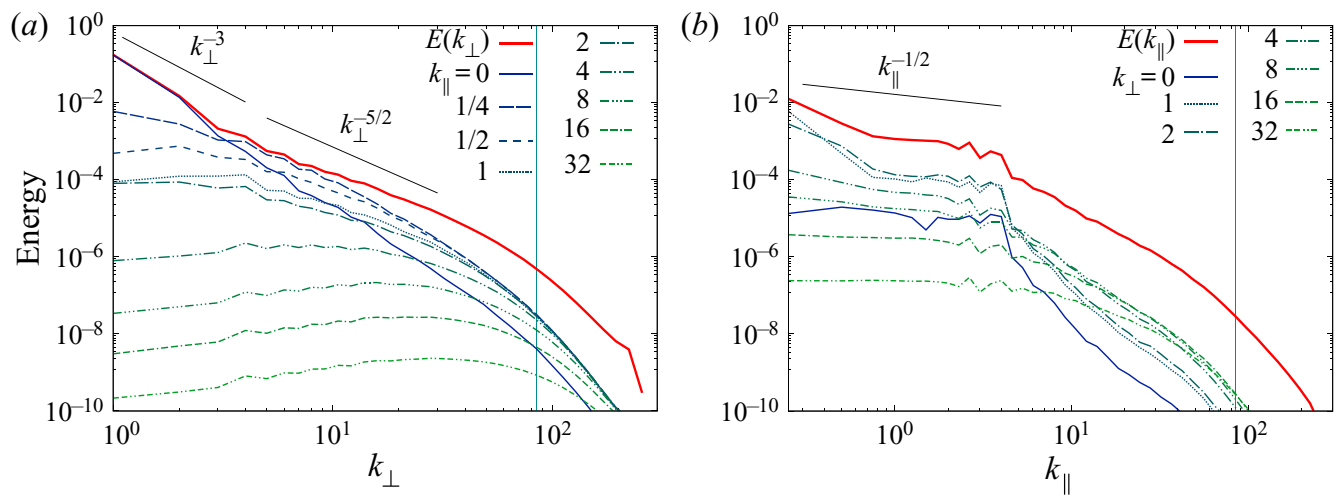

FIGURE 10. Kinetic energy spectra (a) for each $k_{\|}$as a function of $k_{\perp}$ and $(b)$ for each $k_{\perp}$ as a function of $k_{\|}$.
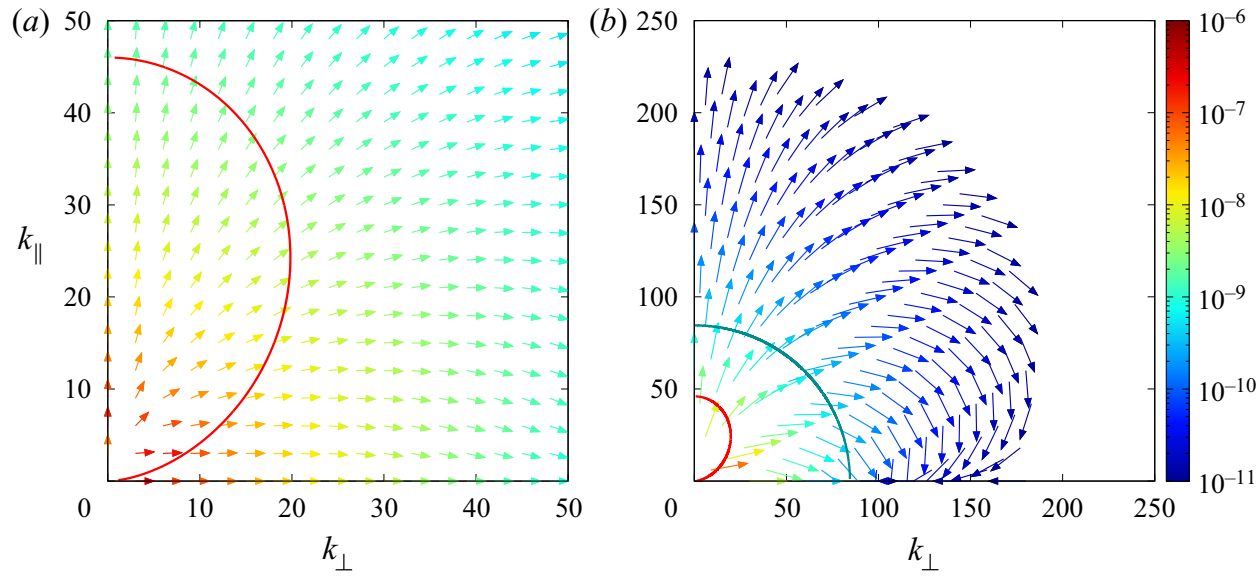

FIGURE 11. Energy-flux vectors where an isotropic external force is applied $(a)$ in the small-wavenumber range and $(b)$ in the whole computational domain. See also the caption to figure 7.

To confirm the consistency of the energy-flux vectors with the commonly used integrated energy fluxes, the integrated fluxes are obtained from the three-dimensional flux vectors and compared with those obtained by (2.5) and (2.6) in figure 12. The isotropic and perpendicular fluxes obtained from the three-dimensional flux vectors are almost equal to those obtained by (2.5) and (2.6) except for the small wavenumbers $k, k_{\perp}<8$. The difference at small wavenumbers comes from the discreteness of the cells and the averaging for $k_{\perp}$ and $k$. The parallel flux obtained from the three-dimensional flux vectors and the corresponding integrated flux are the same within the convergence criterion adopted in the GMRES method. These agreement and disagreements have been seen in the energy flux in HIT (figure 5b). Therefore, the energy-flux vectors are consistent with the integrated energy fluxes, which have been usually used in anisotropic turbulence. 


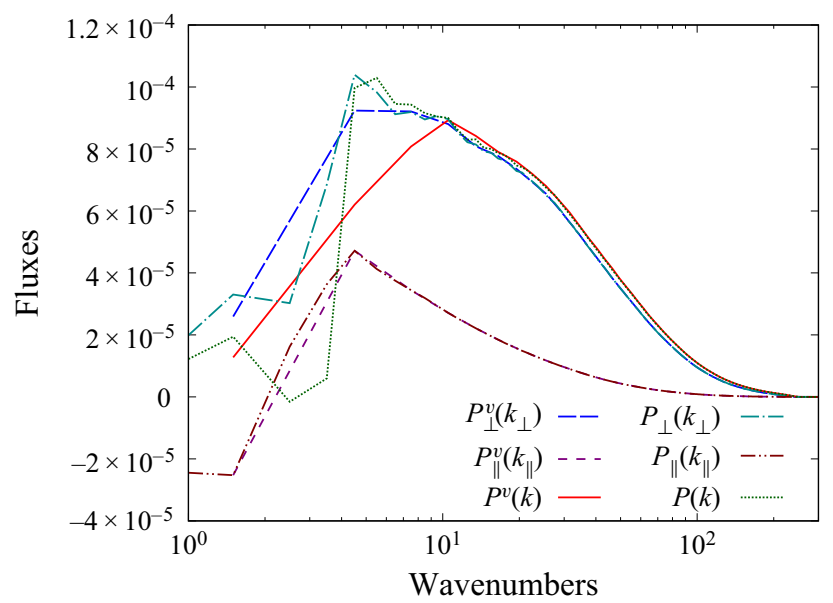

FIGURE 12. Perpendicular, parallel and isotropic integrated fluxes in isotropically forced rotating turbulence. The superscript $v$ denotes the integrated fluxes obtained from the three-dimensional energy-flux vectors. See also the caption to figure 5.

\section{Discussion}

The minimal-norm energy-flux vector successfully shows the local structures of the energy flux both in HIT corresponding to strong turbulence (figure 4) and in anisotropic weak-wave turbulence (figure 7). Thus, one can expect that the minimal-norm vector can be applied also in the buffer range between the weak turbulence range and the strong turbulence range. In contrast to the prediction by the critical balance, however, the energy flux along the wavenumbers at which the linear wave periods and the nonlinear eddy turnover times are comparable is not observed in the present simulations. In fact, the energy flux appears to have a sink in the buffer range.

It is important to clarify the reason for the discrepancy between the numerical result shown in the present paper and the prediction of the weak turbulence theory. To find the reason, the two-dimensional spectrum of energy dissipation rate in the DNS with isotropic external force is shown in figure 13. Note again that the dissipation rate and the energy transfer rate statistically balance at all wavenumbers except for the forced wavenumbers in the ideal statistically steady states. The two-dimensional spectrum is defined as

$$
D\left(k_{\perp}, k_{\|}\right)=\frac{1}{2 \pi k_{\perp}} \frac{1}{\Delta k_{\perp}} \sum_{\boldsymbol{k}_{\perp}^{\prime}}^{\prime} \frac{1}{\Delta k_{\|}} \sum_{k_{z}^{\prime}}^{\prime} v\left(\left|\boldsymbol{k}_{\perp}^{\prime}\right|^{2}+k_{z}^{\prime 2}\right)\left\langle\left|\boldsymbol{u}_{k_{\perp}^{\prime}, k_{z}^{\prime}}\right|^{2}\right\rangle,
$$

where $1 /\left(2 \pi k_{\perp}\right)$ is introduced to easily compare the two-dimensional spectrum with the isotropic one. The dissipation rate is large at the forced wavenumbers and at the wavenumbers of the large-scale columnar vortex which has $k_{\|} \approx 0$. In addition, the dissipation is substantial in the buffer range between the wave-dominant range surrounded by the critical wavenumber and the range of isotropic turbulence where the wavenumber is larger than the Zeman wavenumber.

The energy provided by the external force is transferred to this buffer range by the resonant interactions as seen in figure 11. The critical balance predicts that the energy that reaches the buffer range must start to be isotropised by changing the direction of transfer in this range. However, a sink due to the strong dissipation is observed in this 


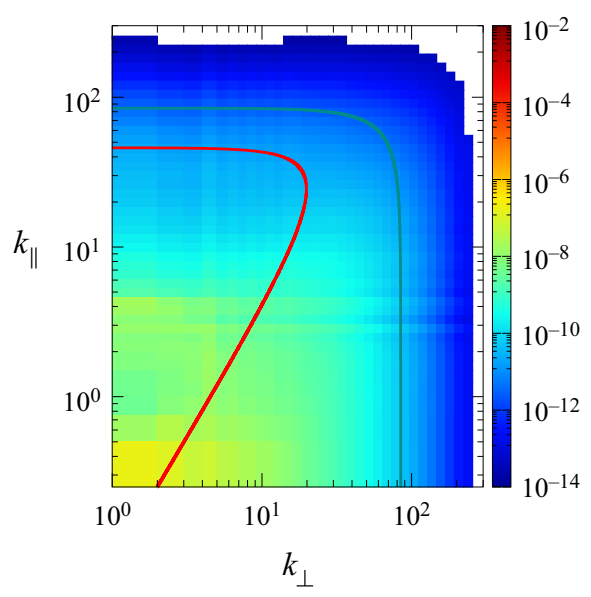

FIGURE 13. Two-dimensional spectrum of energy dissipation rate in rotating turbulence.

range instead of the isotropisation. In this sense, the dissipation plays a dominant role in the buffer range in the present simulations, and the large dissipation there is not considered in the theory of the critical balance. A large range of isotropic turbulence at large wavenumbers is required to determine whether or not the energy is transferred along the critical wavenumbers. The wave-dominant range cannot be small because the resonant interactions are relatively sparse in wavenumber space. Then, high-resolution simulations where both of the wavenumber ranges of weak turbulence and isotropic turbulence are large enough are required.

Let us consider the asymptotic behaviour of the large dissipation which appears in the buffer range in the present simulations at large Reynolds numbers. Suppose that the higher-resolution simulation has a smaller kinematic viscosity without changing the magnitude of the external force and that of the anisotropy, i.e. the system's rotation rate. The buffer range in the higher-resolution simulation remains at almost the same wavenumber range as that in the lower-resolution simulation, because the linear time scales are unchanged and the total energy dissipation rate and hence eddy turnover time are almost the same. On the other hand, the relatively large dissipation in the buffer range should become smaller. Then, some of the energy passes through the dissipation in the buffer range, and the energy flux along the critical wavenumbers that brings isotropisation could be possible.

The critical balance obviously assumes that the dissipation affects only at large wavenumbers outside of the isotropic Kolmogorov turbulence range. In fact, however, the dissipation is not localised; the dissipation spectrum in the inertial subrange of the isotropic Kolmogorov turbulence slowly increases as $k^{1 / 3}$, for example. Thus, a much higher-resolution simulation is required to realise the critical balance. Note that large-eddy simulations and the hyper-viscosity should be used carefully because a change of the dissipation can alter the direction of the energy flux.

The critical balance predicts the isotropisation in the buffer range between the weak turbulence range and the isotropic Kolmogorov turbulence range. Such ranges are characterised by the time scales of dominant physical mechanisms: the linear wave period, the characteristic time of the anisotropic system and the eddy turnover time. When the external force is applied to small perpendicular wavenumbers, $\tau_{w}<\tau_{e} / 3$ in the inertial-wave turbulence range as shown in figures 6 and 7. On the other hand, 
when the external force is applied isotropically to small wavenumbers, the inertial-wave turbulence range goes into the range where $\tau_{w} \sim \tau_{e}$ as shown in figures 10 and 11 . This is mainly due to the high directionality of the energy transfer in inertial-wave turbulence. The wavenumber range of the inertial-wave turbulence depends on the wavenumbers at which the external force is applied as pointed out by Nazarenko \& Schekochihin (2011). The misalignment of the inertial-wave turbulence might indicate the existence of key mechanisms other than the time scales in the energy flux in anisotropic turbulence.

When the energy due to the external force is injected at middle wavenumbers, the energy is expected to cascade to smaller wavenumbers as two-dimensional turbulence and to larger wavenumbers as three-dimensional turbulence (Alexakis \& Biferale 2018). If high-resolution simulations could be performed, such split energy cascades should be observed. In fact, the energy flux towards small $k_{\perp}$ at $k_{\perp}<10$ and $k_{\|} \approx 0$ can be observed in figure 7(a). This two-dimensional inverse cascade suggests that the split energy cascade can be observed using the energy-flux vectors proposed here.

The two ansatzes, the net locality of the energy balance and the efficiency of the energy transfer, are introduced to obtain the minimal-norm energy-flux vector. It is assumed by the former that the local energy transfer is dominant in wavenumber space, and local energy conservation holds. Such locality is essential when the detailed local structures of the energy flux are discussed. It is assumed by the latter that the minimal-norm vector among other possible solutions of local energy conservation is irrotational and excludes local circulations of the energy transfer. This efficiency of the energy transfer is natural because the local circulations make closed loops of the energy fluxes, and do not intrinsically contribute to the net energy flux. Although these ansatzes are introduced, the energy-flux vectors are consistent with the integrated energy fluxes, which have been conventionally used.

\section{Summary}

In anisotropic turbulence, the energy flux in wavenumber space should be considered as a vector at each wavenumber mode, because different kinds of turbulence coexist inhomogeneously at each scale. The integrated energy fluxes that have been used to exhibit anisotropy in the energy transfer cannot reveal detailed local structures of the energy flux, because these integrated fluxes inevitably consist of multiple turbulence ranges. In this work, the minimal-norm energy-flux vector obtained using the Moore-Penrose inverse was proposed to uniquely determine the energy-flux vectors based on two ansatzes: the net locality of the energy balance and the efficiency of the energy transfer. The latter is equivalent to the irrotationality of the energy-flux vectors.

The minimal-norm energy-flux vector is tested in both strongly nonlinear isotropic turbulence and anisotropic weak-wave turbulence. The minimal-norm vectors successfully show the isotropic energy flux that radiates outward in HIT. In rotating turbulence, the minimal-norm flux in the weak turbulence range demonstrates the energy flux parallel to the perpendicular wavenumber axis. Namely, the minimal-norm vectors successfully display energy flux consistent with the energy transfer due to resonant interactions among the inertial waves according to the weak turbulence theory.

On the other hand, the energy flux along the critical wavenumbers predicted by the critical balance was not observed in the buffer range between the weak turbulence range and the isotropic Kolmogorov turbulence range in the rotating turbulence in this paper. The inconsistency of the energy-flux vector proposed here with the critical balance does not indicate the failure of the proposed method but results from the appearance 
of large dissipation in the buffer range. This inconsistency will be resolved when much higher-resolution simulations are performed. In addition, a two-dimensional turbulence such as quasi-geostrophic turbulence is more suitable for obtaining the coexistence of the large-wavenumber ranges of weak-wave turbulence and strong turbulence as well as the buffer range. The energy-flux vector in two-dimensional turbulence will be reported elsewhere.

\section{Acknowledgements}

Numerical computation in this work was carried out at the Yukawa Institute Computer Facility, Kyoto University and the Research Institute for Information Technology, Kyushu University. This work was supported in part by JSPS KAKENHI grant no. 15K17971, no. $16 \mathrm{~K} 05490$, no. $17 \mathrm{H} 02860$, no. $18 \mathrm{~K} 03927$ and no. 19K03677.

\section{Declaration of interests}

The authors report no conflict of interest.

\section{REFERENCES}

ALeXA KIs, A. 2017 Helically decomposed turbulence. J. Fluid Mech. 812, 752-770.

Alexakis, A. \& Biferale, L. 2018 Cascades and transitions in turbulent flows. Phys. Rep. 767-769, $1-101$.

Alexakis, A., Bigot, B., Politano, H. \& Galtier, S. 2007 Anisotropic fluxes and nonlocal interactions in magnetohydrodynamic turbulence. Phys. Rev. E 76, 056313.

Bellet, F., Godeferd, F. S., SCOTT, J. F. \& CAMBon, C. 2006 Wave turbulence in rapidly rotating flows. J. Fluid Mech. 562, 83-121.

Biven, L., NAZARenko, S. V. \& Newell, A. C. 2001 Breakdown of wave turbulence and the onset of intermittency. Phys. Lett. A 280, 28-32.

Biven, L. J., Connaughton, C. \& Newell, A. C. 2003 Structure functions and breakdown criteria for wave turbulence. Physica D 184, 98-113.

Chan, C.-K., Mitra, D. \& Brandenburg, A. 2012 Dynamics of saturated energy condensation in two-dimensional turbulence. Phys. Rev. E 85, 036315.

Clark di Leoni, P., Cobelli, P. J., Mininni, P. D., Dmitruk, P. \& Matthaeus, W. H. 2014 Quantification of the strength of inertial waves in a rotating turbulent flow. Phys. Fluids 26, 035106.

Connaughton, C. \& NAZARENKo, S. 2004 Warm cascades and anomalous scaling in a diffusion model of turbulence. Phys. Rev. Lett. 92, 044501.

Deusebio, E., Boffetta, G., Lindborg, E. \& Musacchio, S. 2014 Dimensional transition in rotating turbulence. Phys. Rev. E 90, 023005.

Dyachenko, S., Newell, A. C., Pushkarev, A. \& Zakharov, V. E. 1992 Optical turbulence: weak turbulence, condensates and collapsing filaments in the nonlinear Schrödinger equation. Physica D $57,96-160$.

Galtier, S. 2003 Weak inertial-wave turbulence theory. Phys. Rev. E 68, 015301.

GALTIER, S. \& BUCHLIN, É. 2010 Nonlinear diffusion equations for anisotropic magnetohydrodynamic turbulence with cross-helicity. Astrophys. J. 722, 1977-1983.

Galtier, S., Nazarenko, S. V., Buchlin, É. \& Thalabard, S. 2019 Nonlinear diffusion models for gravitational wave turbulence. Physica D 390, 84-88.

Ghim, Y.-C., Schekochihin, A. A., Field, A. R., Abel, I. G., Barnes, M., Colyer, G., Cowley, S. C., Parra, F. I., Dunai, D. \& Zoletnik, S. 2013 Experimental signatures of critically balanced turbulence in MAST. Phys. Rev. Lett. 110, 145002.

Goldreich, P. \& SRIDHAR, S. 1995 Toward a theory of interstellar turbulence. II. Strong Alfvénic turbulence. Astrophys. J. 438, 763-775. 
Hasselmann, S., Hasselmann, K., Allender, J. H. \& Barnett, T. P. 1985 Computations and parameterizations of the nonlinear energy transfer in a gravity-wave specturm. Part 2. Parameterizations of the nonlinear energy transfer for application in wave models. J. Phys. Oceanogr. 15, 1378-1391.

Kolmogorov, A. N. 1941 The local structure of turbulence in an incompressible viscous fluid at very large Reynolds numbers. Dokl. Akad. Nauk SSSR 30, 299-303.

KRAICHNAN, R. H. 1959 The structure of isotropic turbulence at very high Reynolds numbers. J. Fluid Mech. 5, 497-543.

KRAICHNAN, R. H. 1971 Inertial-range transfer in two- and three-dimensional turbulence. J. Fluid Mech. 47, 525-535.

LEITH, C. E. 1967 Diffusion approximation to inertial energy transfer in isotropic turbulence. Phys. Fluids 10, 1409-1416.

LindBoRg, E. 2006 The energy cascade in a strongly stratified fluid. J. Fluid Mech. 550, 207-242.

Matthaeus, W. H., Oughton, S. \& Zhou, Y. 2009 Anisotropic magnetohydrodynamic spectral transfer in the diffusion approximation. Phys. Rev. E 79, 035401.

Meyrand, R., Galtier, S. \& Kiyani, K. H. 2016 Direct evidence of the transition from weak to strong magnetohydrodynamic turbulence. Phys. Rev. Lett. 116, 105002.

Meyrand, R., Kiyani, K. H., Gürcan, Ö. D. \& Galtier, S. 2018 Coexistence of weak and strong wave turbulence in incompressible hall magnetohydrodynamics. Phys. Rev. X 8, 031066.

Mininni, P. D., Rosenberg, D. \& Pouquet, A. 2012 Isotropization at small scales of rotating helically driven turbulence. J. Fluid Mech. 699, 263-279.

NAZARENKO, S. 2011 Wave Turbulence. Springer.

NAZARenko, S. V. \& Schekochinin, A. A. 2011 Critical balance in magnetohydrodynamic, rotating and stratified turbulence: towards a universal scaling conjecture. J. Fluid Mech. 677, 134-153.

Newell, A. C., Nazarenko, S. \& Biven, L. 2001 Wave turbulence and intermittency. Physica D 152-153, 520-550.

OhkitAni, K. \& KidA, S. 1992 Triad interactions in a forced turbulence. Phys. Fluids A 4, $794-802$.

Richardson, L. F. \& LynCH, P. 2007 Weather Prediction by Numerical Process, 2nd edn. Cambridge University Press.

ScOTT, R. B. \& ARBIC, B. K. 2007 Spectral energy fluxes in geostrophic turbulence: implications for ocean energetics. J. Phys. Oceanogr. 37, 673-688.

Sharma, M. K., Verma, M. K. \& Chakraborty, S. 2019 Anisotropic energy transfers in rapidly rotating turbulence. Phys. Fluids 31, 085117.

Smith, L. M. \& WALEFFE, F. 1999 Transfer of energy to two-dimensional large scales in forced, rotating three-dimensional turbulence. Phys. Fluids 11, 1608-1622.

TenBarge, J. M. \& Howes, G. G. 2012 Evidence of critical balance in kinetic Alfvén wave turbulence simulations. Phys. Plasmas 19, 055901.

Vallis, G. K., Carnevale, G. F. \& Young, W. R. 1989 Extremal energy properties and construction of stable solutions of the Euler equations. J. Fluid Mech. 207, 133-152.

Vinen, W. F. \& Niemela, J. J. 2002 Quantum turbulence. J. Low Temp. Phys. 128, 167-231.

WALEFFE, F. 1992 The nature of triad interactions in homogeneous turbulence. Phys. Fluids A 4, 350-363.

WAlefFe, F. 1993 Inertial transfers in the helical decomposition. Phys. Fluids A 5, 677-685.

YARom, E. \& Sharon, E. 2014 Experimental observation of steady inertial wave turbulence in deep rotating flows. Nat. Phys. 10, 510-514.

YokоYAма, N. \& TAKAOKA, M. 2014 Identification of a separation wave number between weak and strong turbulence spectra for a vibrating plate. Phys. Rev. E 89, 012909.

YoKoYAma, N. \& TAKAOKA, M. 2017 Hysteretic transitions between quasi-two-dimensional flow and three-dimensional flow in forced rotating turbulence. Phys. Rev. Fluids 2, 092602.

YOKOYAMA, N. \& TAKAOKA, M. 2019 Energy-based analysis and anisotropic spectral distribution of internal gravity waves in strongly stratified turbulence. Phys. Rev. Fluids 4, 104602.

Zakharov, V. E., L'vov, V. S. \& FAlKovich, G. 1992 Kolmogorov Spectra of Turbulence I: Wave Turbulence. Springer. 